\title{
Crystal structure of tribarium arsenide borate, $\mathrm{Ba}_{3} \mathrm{As}_{\mathbf{3}}\left[\mathrm{BO}_{3}\right]$
}

\author{
M. Somer, W. Carrillo-Cabrera, J. Curda, K. Peters and H. G. von Schnering
}

Max-Planck-Institut für Festkörperforschung, Heisenbergstr. 1, D-70569 Stuttgart, Germany

Received October 25, 1996, transferred to 1st update of database ICSD in 1998, CSD-No. 402682

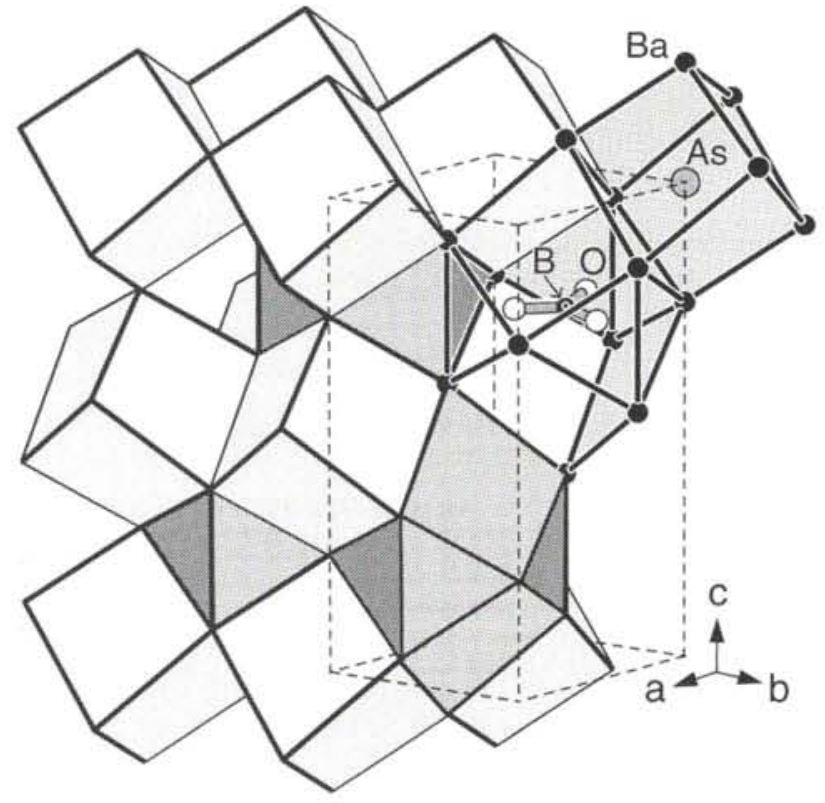

Source of material: $\mathrm{Ba} \mathrm{As}_{\mathrm{A}}\left[\mathrm{BO}_{3}\right]$ is synthesized from a mixture of $\mathrm{Ba}_{3} \mathrm{As}_{2}$ and $\mathrm{Ba}_{3}\left[\mathrm{BO}_{3}\right]_{2}$ (ratio 1:1.2) in a sealed $\mathrm{Nb}$ ampoule at $1550 \mathrm{~K}$.

$\mathrm{Ba} \mathrm{As}_{2}\left[\mathrm{BO}_{3}\right]$ is isostructural to $\left.\mathrm{Ba} 3 \mathrm{P}_{3} \mathrm{BO}_{3}\right]$ (see ref. 1). The crystal structure can be described as a space-filling arrangement of distorted cubes $\mathrm{Ba}$ and Edshammer polyhedra $\mathrm{Ba}_{11}$ which are centered by isolated As atoms and trigonal planar anions $\left[\mathrm{BO}_{3}\right]^{3-}$, respectively $(d(\mathrm{~B}-\mathrm{O})=1.380(11) \AA)$. Note that the $\mathrm{U}_{33}$ parameter for $\mathrm{Ba} 2$ is significantly larger than that found in ref. 1 .
Table 1. Parameters used for the X-ray data collection

Crystal: Wavelength: $\mu$ : Diffractometer: Scan mode:

$\mathrm{T}_{\text {measurement: }}$

$2 \theta_{\max }:$

$\mathbf{N}(h k l)_{\text {urique: }}$

Criterion for $F_{\mathrm{o}}$ :

$\mathrm{N}($ param) refined.

Program:

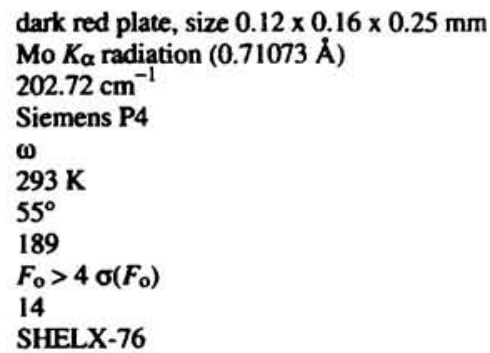

Table 2. Final atomic coordinates and displacement parameters (in $\AA^{2}$ )

\begin{tabular}{llllll}
\hline Atom & Site & $x$ & $y$ & $z$ & $U_{\text {iso }}$ \\
\hline O & $6 h$ & $0.522(2)$ & $1.045(3)$ & $1 / 4$ & $0.019(3)$ \\
B & $2 d$ & $1 / 3$ & $2 / 3$ & $3 / 4$ & $0.021(8)$ \\
\hline
\end{tabular}

AsBBa3O3, hexagonal, $P 63 / m$ (No. 194), $a=5.528(1) \AA$, $c=13.606(2) \AA, V=360.1 \AA^{3}, Z=2, R(F)=0.072, R_{\mathrm{W}}(F)=0.069$.

Table 3. Final atomic coordinates and displacement parameters (in $\AA^{2}$ )

\begin{tabular}{lllllllllll}
\hline Atom & Site & $x$ & $y$ & $z$ & $U_{11}$ & $U_{22}$ & $U_{33}$ & $U_{12}$ & $U_{13}$ & $U_{23}$ \\
\hline $\mathrm{Ba}(1)$ & $4 f$ & $1 / 3$ & $2 / 3$ & $0.0995(2)$ & $0.032(1)$ & $U_{11}$ & $0.033(1)$ & $U_{11} / 2$ & 0 & 0 \\
$\mathrm{Ba}(2)$ & $2 b$ & 0 & 0 & $1 / 4$ & $0.012(1)$ & $U_{11}$ & $0.062(2)$ & $U_{11 / 2}$ & 0 & 0 \\
$\mathrm{As}$ & $2 a$ & 0 & 0 & 0 & $0.037(2)$ & $U_{11}$ & $0.047(4)$ & $U_{11} / 2$ & 0 & 0 \\
\hline
\end{tabular}

\section{References}

1. Somer, M.; Carrillo-Cabrera, W.; Peters, K.; von Schnering, H.G.: Crystal structure of tribarium phosphide borate, $\mathrm{Ba} 3 \mathrm{P}\left(\mathrm{BO}_{3}\right)$. Z. Kristallogr. 210 (1995) 449-450.

2. Sheldrick, G. M.: SHELXL-76, Programs for Crystal Structures Determination. Cambridge, UK 1976. 